\title{
Injury Epidemiology: The Neglected Chapter
}

\author{
Pal R
}

Additional Professor, Department of Community Medicine and Family Medicine

All India Institute of Medical Sciences, Jodhpur-342005, Rajasthan, India

\section{Editorial}

\section{Corresponding Author:}

Dr. Ranabir Pal

Additional Professor

Department of Community Medicine and Family Medicine

All India Institute of Medical Sciences, Jodhpur-342005,

Rajasthan, India

Email: ranabirmon@yahoo.co.in

\section{Abstract}

Injuries are the significant public health problem and posing as the mounting global crisis upsetting families and communities across population levels. We need a multidisciplinary approach embedded in injury surveillance. A sensitization revolution is vital at all the health care levels to gather critical strength of dedicated personnel in the field of research in injury intervention.

\section{Prologue}

In the transition from infectious disease models to chronic disease models, injury epidemiology has been shaped by general developments in epidemiology. It deals with the distribution and determinants of injuries that are entirely predictable and preventable. Epidemiologic research on socio-economic, behavioral, ecological and technological solutions is the call of the day with better understanding within the context of public health. Since the previous century, epidemiologists have focused on reducing the burden of infectious disease with the development of vaccines and antibiotics. This led to a shift in stress on chronic diseases. John Gordon noted similarities to the study of infectious diseases to injury with known patterns and began to view injuries as part of the epidemiologic triad of host, agent, and environment. Initially, the agent of injury was viewed as the object involved (e.g. vehicle, machine part, etc.). Then energy transfer in injury mechanism was well recognized by James Gibson and later refined by William Haddon ${ }^{1}$.

\section{Injury and Accident are not interchangeable words}

"Injuries" are often equated with "accidents" though the latter implies a random nature or unavoidability. In fact most injuries show clear non-random patterns and have identifiable risk factors leading to the observation that injuries does not occur by chance. An accident transpires by chance indicating a random and overpowering unforeseen event, whereas injury is a definable, correctable happening with specific risks for specific events amenable to intervention. Injury is defined as damage in human tissue resulting from exposure to energy delivered in excess of the threshold that human biological systems can tolerate during exposure to various forms of energy. So 'Injuries are not Accidents', is also a simple catchphrase to change civic perception. Injury most often occurs in certain risk groups in a predictable person-time-place distribution, along with identifiable characteristics that are keys to prevent injuries. There are countless domestic, peri-domestic and occupational injuries with varying degrees of quality and quantity with credible downstream disabilities. Globally, amid growing incidence of injury, road traffic injury (RTI) is the leading cause of death in the reproductive age group along with disability and disfigurement.

Injury clearly deserves attention as it occurs as a distinctive stream of events in modern life resulting in bodily damage from sudden exposure to thermal, mechanical, electrical, or chemical energy that kills or disables otherwise healthy people rapidly, unlike other leading causes of death, that are appreciable only after months or years of risk exposure. Injury progresses within seconds of exposure to risk factors, challenging epidemiologic investigations to be at par with other diseases that hinder understanding of continuum of pre-pathogenesis to pathogenesis ${ }^{2}$. 


\section{Let's shed shortage of vision}

Injury continues to grow into a snowballing global crisis, upsetting families and communities across population levels. A good amount of scientific literature has reported on RTIs, though they represent only a tip of iceberg of the injury spectrum. But, the general mindset towards injury completes a vicious cycle as we fail to understand the depth of the problem; thus we do not take any step towards reducing it. We need better perception of the burden of injury, risk factors and injury mechanism from secondary data sources and epidemiological field studies to identify and develop promising design to identify socio-economic costs.

Globally, injuries account for one in eight deaths among males and one in 14 deaths among females. RTIs alone constitute the ninth leading cause of disease burden as measured by the number of associated disability-adjusted life years. By 2020, RTIs are projected to increase in rank to take third place. The gravity of the situation is such that the sum total of mortality due to major killer diseases including Tuberculosis, HIV/AIDS, and Malaria in 2004 was less than half of that of RTI in India ${ }^{3,4}$. Researchers have recommended research guidelines to standardize case definition, and data reporting parameters on traumatic brain injury (TBI) with cumulative hospitalized plus fatal incidence rate 235 per lac, mortality rate 15 per lac and case fatality rate 11 percent ${ }^{5}$.

\section{What is to be done?}

Understanding the principles of injury mechanics and the physical and physiologic responses of the human body to the impact is the fundamental basis of injury science. Injury epidemiology now has to move towards rigorous analytical methods to unearth the underlying injury mechanism to understand the complex array of factors that influence the incidence, severity, and outcomes. Further evaluations of interventions are needed for updating of programs and policies with assessments of cost-effective intervention with effective collaborations between epidemiologists and scientists from the behavioral sciences, sociology, criminology, law, engineering, and biomechanics. Though TBIs is the leading cause of both death and disability among younger segment, its biomechanics is not well understood. The development of effective interventions is dependent on a better understanding of the role of conduct in injury as they are influenced through educational, behavioral, service and legislative approaches to benefit from risk perception, and behavioral responses to safety improvements ${ }^{4}$.

Injury epidemiology describes magnitude of events, risk factors, and the evaluation of intervention programs applied in various environments like occupation, home, roads, transportation, and recreational places as well as internalization of preventive measures like automobile policy, ergonomics, and workplace safety issues, etc. We have to evolve a multidisciplinary approach in the injury surveillance system with dynamic input from the experts in ergonomics, anthropology, industry, health and safety.

\section{William Haddon, Jr. "Father of Injury Epidemiology"}

Following in the footsteps of Gibson in the study of energy transfer, William Haddon looked at injury research as a science. He characterized energy as the "agent" of Injuries and looked at the phases of crashes and linking of phases to epidemiologic triad to identify processes in which interventions could succeed with standard public health methods. He identified the temporal sequence of a crash and examined this sequence: the circumstances surrounding the event prior to the crash occurring, the circumstances involved during the crash, and those involved after the crash. In his perception, the human, vehicular, and environmental factors involved in each stage, resulting in a crash or an injury can help in prevention planning ${ }^{6}$.

\section{Injuries needing special attention}

Sports injuries occur when athletes are exposed under specific risk conditions, at a known time and place with no common operational definition of injury or its severity. There is no set format for data collection across sports. Further, the methodological factors alter the interpretation as to who-where-when-what as well as how much and what is their outcome-to explain why and how injuries occur to identify strategies ${ }^{7}$. There are dramatic shifts in the most disabling workplace injuries; in the US the top five reported were: overexertion, falls on the same level, falls to a lower level, bodily reaction, and being struck by an object. \$52 billion is spent in direct U.S. worker compensation costs for workplace injuries ${ }^{8}$.

\section{Light at the end of tunnel}

Injuries remain a significant public health concern affecting all age groups, needing continual focus on research and prevention. Reduction in injury and their costs to families and communities are possible but this needs support, collaboration, and partnering at the political level starting from the perceptions of injury as a public health problem. Injury epidemiologists are on the front lines of identifying who are at risk for what kind of injuries, in order to provide the rationale for interventions. Efforts are required in conducting massive collaborative field data collection to index the leading causes and related costs of the most disabling injuries and what they cost to national economy. As people switch jobs, there is change of risk profiles, similarly for contingent workers and immigrant workers, these are not well accounted for. There has been increasing attention with the efforts of the public health agencies to reduce injury initiated counting and calculating rates, identifying causes and measuring outcomes of interventions ${ }^{9}$.

\section{Hidden agenda}

In South-east Asian countries, the health teaching-learning are all done in a disconnected manner so students fail to learn that the risk factors produce a trail of events leading to injury and thus missing the point that early interventions in the natural history can affect the prognosis. Even when 
non-communicable diseases are taught, rarely do we note any methodical approach to injury science based on the epidemiological triad; even the epidemiologists are unaware. Further, we have a casual approach with the understanding that injury care is the domain of Orthopedics, Neurosurgery and other specialist disciplines. A much awaited resolution is needed to bring together within systematic training not only all the streams of health sciences but also all schools of medicine.

\section{Hope for the best}

We need sensitization with the introduction of surveillance to identify risk factors of injury at all levels of health care. We have to gain a better understanding of the theories underlying prevention and precisely map out their application; develop an appreciation of the process of priority setting in injury, the design and implementation of injury interventions with time bound evaluations. Health education should reorient to internalize that injury is a multi-disciplinary arena and needs a holistic approach; we cannot tackle this public health problem only by approaches in injury data sources, surveillance systems or research methods. The shortage of trained manpower at all levels of injury science has to be solved by a global education platform. Further, laws related to injury need to be revised as injury is treated as a medico-legal case in many countries (including India) that leads to underreporting even in cases of serious injuries. Lastly, more dedicated personnel are needed in the field of operation research in injury epidemiology that is a well-known bottle neck in the intervention.

\section{Conflict of Interests}

The authors have no conflict of interest arising from the study.

\section{References}

1. Sleet D A, Moffett D B. Framing the problem: injuries and public health. Fam Community Health 2009; 32(2):88-97.

2. CCEB: Injury Epidemiology [Internet]. [cited 2012 Oct 4]. Available from: http://www.cceb.upenn.edu/research/ injury-epi.php

3. Mondal P, Kumar A, Bhangale U D, Dinesh Tyagi D. A Silent Tsunami on Indian Road: A Comprehensive Analysis of Epidemiological Aspects of Road Traffic Accidents. Br J Med Med Res 2011; 1(1): 14-23.

4. MacKenzie E J. Epidemiology of injuries: current trends and future challenges. Epidemiol Rev 2000; 22(1):112-9.

5. Tagliaferri F, Compagnone C, Korsic M, Servadei F, Kraus J. A systematic review of brain injury epidemiology in Europe. Acta Neurochir (Wien) 2006; 148(3):255-68; discussion 268.

6. Songer T. History of Injury Epidemiology. [online] [cited 2012 Oct 15] Available from: www.pitt.edu/ epi2670/ injuryhistory/History2009.pdf
7. Phillips L H. Sports injury incidence. Br J Sports Med 2000; 34: 133-6.

8. Bennett A. Slips, Trips, and Falls [Internet]. The Responsibility Project by Liberty Mutual. 2010 [cited 2012 Oct 4]. Available from: http://responsibilityproject.libertymutual.com/articles/slips-trips-and-falls 9. Cummings P, Koepsell T D, Mueller B A. Methodological challenges in injury epidemiology and injury prevention research. Annu Rev Public Health 1995; 16: 381-400.

\begin{tabular}{|l|l|}
\hline \multicolumn{2}{|c|}{ Article Information } \\
\hline \multicolumn{2}{|c|}{ Article history } \\
\hline Received & 10 October 2012 \\
Received in revised form & 15 October 2012 \\
Accepted & 30 November 2012 \\
\hline
\end{tabular}

